\title{
10063-36 Impact of blood volume changes within the human skin on the diffuse reflectance measurements in visible and NIR spectral ranges \\ SPIE. ${ }^{1 O p t o-E l e c t r o n i c s ~ a n d ~ M e a s u r e m e n t ~ T e c h n i q u e s, ~ F a c u l t y ~ o f ~ I n f o r m a t i o n ~ T e c h n o l o g y ~ a n d ~ E l e c t r i c a l ~ E n g i n e e r i n g, ~ U n i v e r s i t y ~ o f ~ O u l u ~, ~ E r k k i ~ K o i s o-~}$ Kanttilankatu 3, Oulu, 90570, Finland \\ ${ }^{2}$ Computer Graphics Group, Department of Computer Science, Yale, University, New Haven, CT 06520, USA \\ ${ }^{3}$ Biomedical Photonics Instrumentation Group, Scientific-Educational Center "Biomedical Engineering", Oryol State University, Komsomolskaya b. 95, Oryol, 302026, Russia
}

\section{INTRODUCTION}

At present, measurements of diffuse light reflectance from different biological tissues in vivo and in vitro are widely employed as a measurement procedure. The recording of the diffuse reflectance is used in such diagnostical technologies as steady-state diffuse reflectance spectroscopy, diffusing-wave spectroscopy, diffuse correlation spectroscopy, pulse oximetry etc $[1,2]$. In all cases of using optical measurements to assess parameters of a biological object in vivo and in situ, it is important to know the actual sampling volume (SV) of the biological tissue. An alteration of the blood volume fraction, as well as oxygen saturation level (in case of measurements beyond of isobestic points of $\mathrm{Hb} / \mathrm{HbO} 2$ pair), has strong impact on the geometrical parameters of sampling volume [3].

The aim of this work was to study the effect of sampling volume pulsation of diffuse reflectance measurements, which arises because of blood volume fraction pulsation as well as pulsation of blood saturation.

\section{METHODS}

In this study, the diffuse reflectance measurements of the QR400-7SR (Ocean Optics, Inc) probe sampling volume were modelled and analyzed. The probe has 6 emitting fibers and one collecting fiber (Fig. 1).

A CUDA-based Monte-Carlo distributed computing platform was used for routine simulation of SV and skin reflectance spectra, as well as their variations associated with

\section{METHODS}

Saturation pulsation was modeled by alteration of saturation in the range of $85 \%-95 \%$. Modulation of the blood volume fraction, as well as blood saturation, were completed using the photoplethysmography function (Fig. 2).
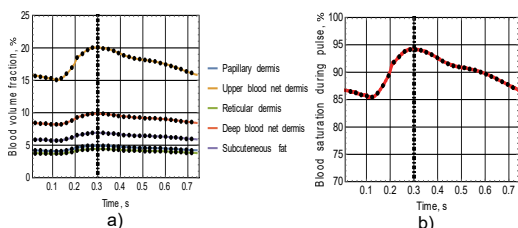
Time,s a) Fig. 2 Modeling of alteration of blood volume fraction (a) and saturation (b) during pulse

The calculations were carried out for the following wavelengths: $434 \mathrm{~nm}, 450 \mathrm{~nm}, 480 \mathrm{~nm}, 500 \mathrm{~nm}, 545 \mathrm{~nm}$, $560 \mathrm{~nm}, 660 \mathrm{~nm}, 800 \mathrm{~nm}$.

\section{RESULTS}

The results of modelling of the sampling volume for the plane perpendicular to the line connecting the centers of the receiving and transmitting fiber are presented in Fig. 3,4. The mean calculated sampling volumes for different wavelengths of probing radiation are presented in Tab. 1 . Relative increment of the pulsatile calculated sampling volume for different wavelength during on pulse period are presented in Fig. 5 .

Table 1 Mean calculated sampling volume

\begin{tabular}{|l|l|l|l|l|l|l|l|l|}
$\lambda, \mathrm{nm}$ & 434 & 450 & 480 & 500 & 545 & 560 & 660 & 800 \\
\hline
\end{tabular} \begin{tabular}{|c|c|c|c|c|c|c|c|c|}
\hline $\mathrm{SV}, \mathrm{mm}^{3}$ & 0.37 & 0.77 & 0.48 & 1.80 & 1.05 & 1.30 & 28.0 & 18.5 \\
\hline
\end{tabular} physiological changes in the human skin $[4,5,6]$
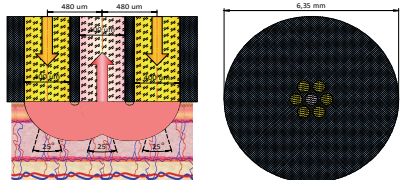

Fig. 1 The schema of measurements a human skin by the probe QR400-7SR (Ocean Optics, Inc)

In the used model, the absorption coefficients of each layer take into account the concentration of blood $C_{\text {blood }}$ in various vascular beds, oxygen saturation $S$, water content $C_{H 2 O}$ and melanin fraction $C_{m e /}$ as it is defined in $[5,6]$.

Blood pulsation was modelled by relative alteration of blood volume fraction of each skin layer the range of $85 \%-100 \%$.

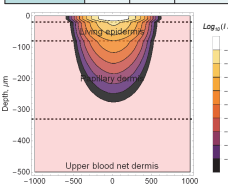
a) $434 \mathrm{~nm}$

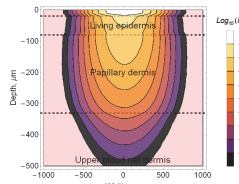

c) $480 \mathrm{~nm}$

sampling volume

Fig. 3 Calculated sampling volume of diffuse reflectance
measurements for the modelled optical probe for several wavelengths of blue optical range

b) $450 \mathrm{~nm}$

d) $500 \mathrm{~nm}$
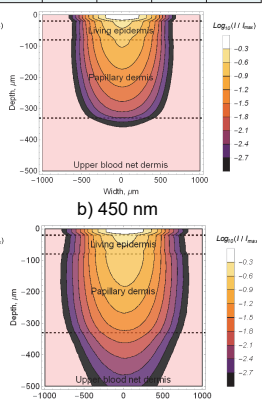

University of Oulu, Optoelectronics and measurement techniques laboratory - www.biophotonics.fi

RESULTS

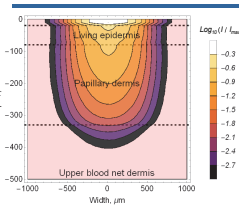

a) $545 \mathrm{~nm}$

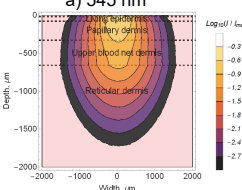

c) $660 \mathrm{~nm}$

Fig. 4 Calculated sampling volume of diffuse reflectance measurements for the modelled optical probe for several wavelengths green, yellow, red and NIR optical ranges

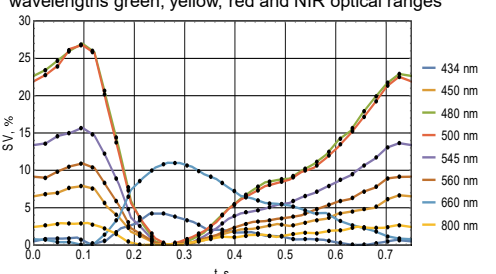

Fig. 5 Relative increment of calculated sampling volume for different wavelengths during on pulse period

\section{CONCLUSIONS}

We have considered changes in the volume of blood and oxygen saturation caused by a pulse wave and their influence on the diffuse reflectance spectra in the visible/NIR spectral range. A CUDA-based Monte-Carlo model was used for routine simulation of detector depth sensitivity (sampling volume) and skin spectra, and their variations associated with physiological changes in the human skin. The results are of particular interest for pulse oximetry, photoplethysmography, Doppler flowmetry, reflectance spectroscopy.

REFERENCES

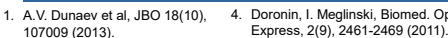

2. A.V. Dunaev et al, Med Eng Phys. 5. G.I. Petrov et al., Biomed. Opt. Express, $37(6), 574-583(2015) . \quad 3(9), 2154-2161(2012)$. I. Meglinski et al, Med. Biol. Eng. 6. I. Meglinski et al, Computational Biophysics
Comput,. 39, 44-50 (2001).
of the Skin, Ed. B. Querleux, Singapore,
Chapter 2 (2014).

Dynamics and Fluctuations in Biomedical Photonics XIV, edited by Valery V. Tuchin, Kirill V. Larin, Martin J. Leahy, Ruikang K. Wang, Proc. of SPIE Vol. 10063, 1006312 · (c) 2017 SPIE

CCC code: $1605-7422 / 17 / \$ 18 \cdot$ doi: $10.1117 / 12.2250855$ 